\title{
Invariant tori for Hamiltonian PDE
}

\author{
By Walter CRAig \\ Department of Mathematics and Statistics \\ McMaster University \\ Hamilton, Ontario L8S 4K1, Canada
}

Many of the central equations of mathematical physics, the nonlinear wave equation, the nonlinear Schrödinger equation, the Euler equations for free surfaces, can be posed as Hamiltonian systems with infinitely many degrees of freedom. In a neighborhood of an equilibrium, the linearized equations are those of a harmonic oscillator and thus solutions exhibit periodic and quasi-periodic motion. To construct solutions of the same nature for the nonlinear partial differential equations $(\mathrm{PDE}) \mathrm{s}$ is a small divisor problem in general. This article gives an overview of some of the techniques and results of KAM-like methods for PDE, which have been developed to address the analysis of this problem.

Keywords: Hamiltonian partial differential equations, dynamical systems

\section{Introduction}

A Hamiltonian partial differential equation is an evolution equation whose initial value problem takes the form

$$
\partial_{t} v=\operatorname{Jgrad}_{v} H(v), \quad v(x, 0)=v_{0}(x),
$$

where $v \in \mathcal{H}$ which is a Hilbert space playing the rôle of the phase space, which has a symplectic form given by

$$
\omega(X, Y)=\left\langle X, J^{-1} Y\right\rangle_{\mathcal{H}}, \quad J^{T}=-J .
$$

The flow of this dynamical system, if it exists on a neighborhood of $v_{0}(x)$, is denoted by

$$
v(x, t)=\varphi_{t}\left(v_{0}(x)\right)
$$

tracing a curve in $\mathcal{H}$ through $v_{0}$. Our aim is to describe some analytic results constructing special compact invariant sets for this flow in the phase space $\mathcal{H}$. The outline of this article are as follows:

Section 2. Hamiltonian PDE - principal examples

Section 3. Invariant tori - a variational problem

Section 4. Estimates of the linearized problem 


\section{Principal examples}

(a) nonlinear wave equations

This is a model for a class of nonlinear wave equations for which the function $u(x, t)$ represents a scalar field. We seek solutions of the equation

$$
\partial_{t}^{2} u-\Delta u+g(x, u)=0,
$$

satisfying a boundary condition on a domain $\Omega \subseteq \mathbb{R}^{d}$, which could be Dirichlet or Neumann conditions. More conveniently one often poses periodic boundary conditions on a torus, which is to say that $\Omega=\mathbb{T}^{d}=\mathbb{R}^{d} / \Gamma$ for a given period lattice $\Gamma$. This is what we will do for the most part of this article. The Hamiltonian functional for problem (2.1) is given by

$$
H(u, p)=\int_{\mathbb{T}^{d}} \frac{1}{2} p^{2}+\frac{1}{2}|\nabla u|^{2}+G(x, u) d x,
$$

with respect to which the equations (2.1) can be rewritten as

$$
\begin{aligned}
\partial_{t} u & =p=\operatorname{grad}_{p} H(u, p) \\
\partial_{t} p & =\Delta u-\partial_{u} G(x, u)=-\operatorname{grad}_{u} H(u, p),
\end{aligned}
$$

where $g(x, \cdot)=\partial_{u} G(x, \cdot)$. This is to say that the system has the form

$$
\partial_{t}\left(\begin{array}{l}
u \\
p
\end{array}\right)=\left(\begin{array}{cc}
0 & I \\
-I & 0
\end{array}\right)\left(\begin{array}{l}
\operatorname{grad}_{u} H(u, p) \\
\operatorname{grad}_{p} H(u, p)
\end{array}\right),
$$

and the symplectic form is given by

$$
\omega(X, Y)=\int_{\mathbb{T}^{d}} X_{2}(x) Y_{1}(x)-X_{1}(x) Y_{2}(x) d x
$$

for two vector fields $X=\left(X_{1}, X_{2}\right), Y=\left(Y_{1}, Y_{2}\right) \in \mathcal{H}$. Recognizing the canonical form for

$$
J=\left(\begin{array}{cc}
0 & I \\
-I & 0
\end{array}\right) .
$$

we say that this system is posed in Darboux coordinates. Assume that the nonlinear term $G(x, u)$ is sufficiently smooth, and expand in its Taylor series around $(u, p)=0$;

$$
G(x, u)=\frac{1}{2} g_{1}(x) u^{2}+\frac{1}{3} g_{2}(x) u^{3}+\ldots
$$

then the point $(u, p)=0$ is a stationary point for the dynamical system (2.3), and an expression for the Hamiltonian reflecting this expansion about this point is given by

$$
H=H^{(2)}+H^{(3)}+\cdots
$$

An elegant way to linearize this problem about zero is to truncate the Hamiltonian at quadratic order. In doing so we obtain

$$
\begin{aligned}
H^{(2)} & =\int_{\mathbb{T}^{d}} \frac{1}{2} p^{2}+\frac{1}{2}|\nabla u|^{2}+\frac{1}{2} g_{1}(x) u^{2} d x \\
& =\sum_{k \in \mathbb{Z}^{d}} \frac{1}{2}|p(k)|^{2}+\frac{1}{2} \omega(k)^{2}|u(k)|^{2},
\end{aligned}
$$


where the coefficients $(u(k), p(k)$ are the generalized Fourier coefficients of an eigenfunction expansion

$$
\left(\begin{array}{l}
u(x) \\
p(x)
\end{array}\right)=\sum_{k \in \mathbb{Z}^{d}}\left(\begin{array}{l}
u(k) \\
p(k)
\end{array}\right) \psi_{k}(x)
$$

and where the eigenfunction, eigenvalue pairs $\left(\psi_{k}(x), \omega^{2}(k)\right)$ satisfy the problem

$$
L\left(g_{1}\right) \psi_{k}=\left(-\Delta+g_{1}(x)\right) \psi_{k}=\omega(k)^{2} \psi_{k} .
$$

This is evidently a harmonic oscillator, with frequencies $\{\omega(k)\}$, for $k \in \mathbb{Z}^{d}$.

All solutions of the linearized equations

$$
\partial_{t}\left(\begin{array}{l}
u \\
p
\end{array}\right)=\left(\begin{array}{cc}
0 & I \\
-I & 0
\end{array}\right)\left(\begin{array}{l}
\operatorname{grad}_{u} H^{(2)}(u, p) \\
\operatorname{grad}_{p} H^{(2)}(u, p)
\end{array}\right),
$$

are given by this eigenfunction expansion for the linear flow (for reasons of simplicity of the discussion we have not pursued cases in which some eigenvalues of (2.7) may be negative, $\left.\omega^{2}(k)<0\right)$;

$$
\begin{aligned}
\left(\begin{array}{l}
u(x, t) \\
p(x, t)
\end{array}\right) & =\sum_{k \in \mathbb{Z}^{d}} \psi_{k}(x)\left(\begin{array}{cc}
\cos (\xi(k, t)) & \frac{1}{\omega(k)} \sin (\xi(k, t)) \\
-\omega(k) \sin (\xi(k, t)) & \cos (\xi(k, t))
\end{array}\right)\left(\begin{array}{c}
u_{0}(k) \\
p_{0}(k)
\end{array}\right) \\
& =\Phi_{t}\left(\begin{array}{l}
u_{0}(x) \\
p_{0}(x)
\end{array}\right) .
\end{aligned}
$$

In this expression, the phases are given by

$$
\xi(k, t)=\omega(k) t .
$$

The simple facts about the flow (2.9) are that

(1) the Hamiltonian $H^{(2)}$ is preserved by the evolution;

$$
H^{(2)}\left(\Phi_{t}(v)\right)=H^{(2)}(v) .
$$

(2) Furthermore, the action functionals are preserved by the flow, where the action is defined by;

$$
\begin{aligned}
I_{k}(u, p) & =\frac{1}{2}\left(\omega(k)|u(k)|^{2}+\frac{1}{\omega(k)}|p(k)|^{2}\right), \\
I_{k}\left(\Phi_{t}(v)\right) & =I_{k}(v) ;
\end{aligned}
$$

(3) The phases evolve linearly in time, as in (2.10). Hence all solutions are either periodic in time, quasiperiodic in time, or at least almost periodic in time. Namely, the orbit $(u(x, t), p(x, t))=\Phi_{t}\left(u_{0}(x), p_{0}(x)\right)$ of $(2.8)$ is periodic when each of the frequencies $\omega\left(k_{j}\right)$ for which at least one of the Fourier coefficients $\left(u_{0}\left(k_{j}\right), p_{0}\left(k_{j}\right)\right)$ is nonzero (the active frequencies) is an integer multiple of a basic frequency $\omega_{0}$;

$$
\omega\left(k_{j}\right)=\ell_{j} \omega_{0}, \quad \ell_{j} \in \mathbb{Z} .
$$

The orbit $\Phi_{t}\left(u_{0}(x), p_{0}(x)\right)$ is quasiperiodic with a $m$-dimensional frequency base if there is a $m$-dimensional frequency vector $\omega_{0}=\left(\omega_{0}(1), \ldots \omega_{0}(m)\right) \in \mathbb{R}^{m}$ such that the active frequencies satisfy

$$
\omega\left(k_{j}\right)=\left\langle\ell_{j}, \omega_{0}\right\rangle, \quad \ell_{j} \in \mathbb{Z}^{m} .
$$


That is, the linear span of the set $\left\{\omega\left(k_{j}\right)\right\}$ over the rationals $\mathbb{Q}$ has dimension $m$. A solution $(u(x, t), p(x, t))$ is almost periodic if no finite number of base frequencies suffices.

These facts motivate a number of basic questions regarding the nonlinear problem (2.3). Namely

(1) whether some solutions of the nonlinear problem have the same properties of periodicity, quasiperiodicity, or almost periodicity as the solutions of the linearized problem. This question is in the area of KAM theory, the study of invariant tori for such Hamiltonian systems.

(2) Whether all solutions with initial data $v_{0}(x) \in \mathcal{H}$ remain in the function space $\mathcal{H}$ for all time $t \in \mathbb{R}$, which is the question of (global) well posedness; whether for any $\delta>0$ there exists a $\varepsilon>0$ such that for $v(x) \in B_{\varepsilon}(0)$ then $\varphi_{t}(v) \in B_{\delta}(0)$ for all $t \in \mathbb{R}$, which is a question of stability; or whether the action variables $\left\{I_{k}\right\}$ vary by controlled amounts over long time intervals. More precisely,

$$
\left|I_{k}\left(\varphi_{t}(v)\right)-I_{k}(v)\right|<\varepsilon^{\alpha}
$$

over time intervals

$$
|t|<T(\varepsilon) \sim \exp \left(\frac{1}{\varepsilon^{\beta}}\right),
$$

which is known as a Nekhoroshov stability result.

(3) Whether there are lower bounds on the growth of the action functionals, or on higher Sobolev norms of the solutions of (2.3). In finite dimensional Hamiltonian systems this phenomenon is known as Arnold diffusion.

\section{(b) nonlinear Schrödinger equations}

The second example of a Hamltonian PDE that we address is the nonlinear Schrödinger equation, which appears in the form

$$
i \partial_{t} u-\frac{1}{2} \Delta u+Q(x, u, \bar{u})=0
$$

where we take $x \in \mathbb{T}^{d}$ representing periodic boundary conditions. One could also take either Dirichlet or Neumann, or other self-adjoint boundary conditions on a domain $\Omega \subseteq \mathbb{R}^{d}$. Setting initial data $u(x, 0)=u_{0}(x) \in \mathcal{H}$, we suppose that we can solve the initial value problem, and we denote the flow of this dynamical system by

$$
u(x, t)=\varphi_{t}\left(u_{0}(x)\right) .
$$

The Hamiltonian is given by the expression

$$
H(u)=\int_{\mathbb{T}^{d}} \frac{1}{2}|\nabla u|^{2}+G(x, u, \bar{u}) d x
$$

where the nonlinearity $G$ is asked to satisfy two conditions; that $\partial_{\bar{u}} G=Q$, and the complex function $G(x, z, w)$ is real valued whenever $w=\bar{z}$. Then $(2.12)$ can be rewritten as

$$
\partial_{t} u=i \operatorname{grad}_{\bar{u}} H(u),
$$

where $J=i I$ is the canonical form of complex symplectic coordinates. 


\section{(c) Korteweg deVries equations}

A third example of a class of Hamiltonian PDE is the Korteweg deVries equation, whose original application was to waves in the free surface of a fluid. In general form this is

$$
\partial_{t} q=\frac{1}{6} \partial_{x}^{3} q-\partial_{x}\left(\partial_{q} G(x, q)\right),
$$

where one takes $x \in \mathbb{R}^{1} / \Gamma=\mathbb{T}^{1}$. The Hamiltonian is given by

$$
H(q)=\int_{\mathbb{T}^{1}} \frac{1}{12}\left(\partial_{x} q\right)^{2}+G(x, q) d x
$$

and the symplectic form is a non-classical one, given by

$$
\omega(X, Y)=\int_{\mathbb{T}^{1}}\left(\partial_{x}^{-1} X(x)\right) Y(x) d x
$$

which is to say that the symplectic form is given in terms of the operator $J=-\partial_{x}$.

\section{(d) large amplitude long waves in an interface}

The equations of motion of a free interface between two immiscible fluids of different densities, which are acted on by gravity, can be described as a Hamiltonian PDE. Suppose that there is a fixed flat bottom boundary at a finite depth $\{y=$ $-h<0\}$, a top boundary at $\left\{y=+h_{1}>0\right\}$ which is a rigid lid, and a sharp interface between the two fluids given by $\{y=\eta(x, t)\}$. Denote the acceleration of gravity by $g$, the density of the lower fluid by $\rho$, and that of the upper fluid by $\rho_{1}<\rho$. In both of the upper and lower fluid regions the velocity field of the fluid is given as a potential flow, namely

$$
\mathbf{u}_{1}=\nabla \varphi_{1} \text { for } \eta(x, t)<y<h_{1}, \quad \mathbf{u}=\nabla \varphi \text { for }-h<y<\eta(x, t) .
$$

Both $\varphi$ and $\varphi_{1}$ are harmonic functions in their respective fluid regions, and they satisfy Neumann boundary conditions on the two rigid boundaries. Such situations occur in the laboratory, and under certain conditions these equations model the behavior of a sharp interface in the ocean between two water layers of different temperatures, such as occurs in the tropics (called a thermocline layer), or between two layers of different salinity (a pycnocline layer), such as occurs in deep fjords subject to large freshwater sources, or on the Mediterranean side of the Gibralter straits. It was discovered by Benjamin \& Bridges [BB97] that this can be written as a Hamiltonian system, in terms of the canonical variables $(\eta(x), \xi(x))$ where one defines $\xi(x)=\rho \varphi(x, \eta(x))-\rho_{1} \varphi_{1}(x, \eta(x))$. A systematic study of the long wave scaling limits of the resulting system of equations is presented in [CGK03, CGK04]. Perhaps the most interesting limiting system of equations when the slope of the interface is of order $\mathcal{O}(\varepsilon)$, without assuming that the actual interface displacement is small. Changing variables through the transformation

$$
u(x)=\partial_{x} \xi(x)
$$

the resulting equations for the interface take the form

$$
\partial_{t}\left(\begin{array}{l}
\eta \\
u
\end{array}\right)=\left(\begin{array}{cc}
0 & -\partial_{x} \\
-\partial_{x} & 0
\end{array}\right)\left(\begin{array}{c}
\operatorname{grad}_{\eta} H \\
\operatorname{grad}_{u} H
\end{array}\right) .
$$


This is evidently a Hamiltonian system with symplectic form given by the expression

$$
\omega(X, Y)=\int\left(\partial_{x}^{-1} X_{1}\right) Y_{2}+\left(\partial_{x}^{-1} X_{2}\right) Y_{1} d x
$$

which is the same as that occurring for the more well known Boussinesq system. In the present case the Hamiltonian functional is given by the expression

$$
\begin{aligned}
H(\eta, u)= & \int \frac{1}{2} R_{0}(\eta) u^{2}+\frac{1}{2} g\left(\rho-\rho_{1}\right) \eta^{2} \\
& \left.\left.+R_{1}(\eta)(\partial)_{x} u\right)^{2}+\frac{1}{4} R_{2}(\eta) \partial_{x} \eta\left(\partial_{x} u^{2}\right)+R_{3}(\eta)(\partial)_{x} \eta\right)^{2} u^{2} d x
\end{aligned}
$$

The nonlinear coefficients of this integrand are rational functions in $\eta$, which are precisely

$$
\begin{aligned}
& R_{0}(\eta)=\frac{(h+\eta)\left(h_{1}-\eta\right)}{\rho_{1}(h+\eta)+\rho\left(h_{1}-\eta\right)} \\
& R_{1}(\eta)=-\frac{1}{3} \frac{(h+\eta)^{2}\left(h_{1}-\eta\right)^{2}\left[\rho_{1}\left(h_{1}-\eta\right)+\rho(h+\eta)\right]}{\left[\rho_{1}(h+\eta)+\rho\left(h_{1}-\eta\right)\right]^{2}} \\
& R_{2}(\eta)=-\frac{1}{3} \rho \rho_{1} \frac{\left(h+h_{1}\right)(h+\eta)\left(h_{1}-\eta\right)\left[\left(h_{1}-\eta\right)^{2}-(h+\eta)^{2}\right]}{\left[\rho_{1}(h+\eta)+\rho\left(h_{1}-\eta\right)\right]^{3}} \\
& R_{3}(\eta)=-\frac{1}{3} \rho \rho_{1} \frac{\left(h+h_{1}\right)^{3}\left[\rho_{1}(h+\eta)^{3}+\rho\left(h_{1}-\eta\right)^{3}\right]}{\left[\rho_{1}(h+\eta)+\rho\left(h_{1}-\eta\right)\right]^{4}} .
\end{aligned}
$$

One can view the first as the nonlinear propagation velocity, and the other three as coefficients of nonlinear dispersion. This system and its derivation are discussed in [CGK04].

\section{(e) free surface water waves}

The water waves problem in $d$-dimensions concerns the dynamics of a free surface $\left\{y=\eta(x, t): x \in \mathbb{T}^{d-1}\right\}$ bounding a fluid region in which the fluid velocity field is given by a potential flow;

$$
\mathbf{u}=\nabla \varphi, \quad \Delta \varphi=0
$$

The potential satisfies Neumann boundary conditions on the bottom boundary of the fluid region $\{y=-h\}$, and as above the fluid has gravity as a restoring force. Given a fluid domain $S(\eta)$ fixed by $\eta(x)$, we parametrize the set of harmonic functions on $S(\eta)$ which satisfy Neumann bottom boundary conditions by their top boundary values $\xi(x)=\varphi(x, \eta(x))$. It was shown by Zakharov in a classical paper [Z68] that the full Euler's equations with a free surface are equivalent to the system

$$
\partial_{t}\left(\begin{array}{l}
\eta \\
\xi
\end{array}\right)=\left(\begin{array}{cc}
0 & I \\
-I & 0
\end{array}\right)\left(\begin{array}{l}
\operatorname{grad}_{\eta} H(\eta, \xi) \\
\operatorname{grad}_{\xi} H(\eta, \xi)
\end{array}\right)
$$

where the Hamiltonian is given by

$$
H(\eta, \xi)=\int \frac{1}{2} \xi G(\eta) \xi+\frac{1}{2} g \eta^{2} d x .
$$


This particular version of the problem is given in Craig \& Sulem [CS93]. The Dirichlet-Neumann operator $G(\eta)$ is an integral operator on the free surface, which solves the following harmonic extension problem:

$$
\begin{aligned}
\xi(x) & \mapsto \varphi(x, y) \quad \text { harmonic extension } \\
& \mapsto N \cdot \nabla \varphi(x, \eta(x)) d x \quad \text { normal derivative } \\
& :=G(\eta) \xi(x) d x
\end{aligned}
$$

Notice that the equations of motion (2.21) involve the gradient of the Dirichlet integral with respect to variations of the domain through an expression for the quantity $\delta_{\eta} G(\eta) \xi$. This question was discussed by Hadamard [H10], and was anticipated by him to be relevant to the study of free surface water waves in [H16].

\section{A variational formulation}

Consider the mapping of a $m$-dimensional torus $\mathbb{T}^{m}$ into our phase space $\mathcal{H}$;

$$
S(\xi): \mathbb{T}^{m} \mapsto \mathcal{H}
$$

which will be flow invariant under the evolution of a Hamiltonian PDE (1.1) in the sense that for some frequency vector $\Omega \in \mathbb{R}^{m}$,

$$
S(\xi+t \Omega)=\varphi_{t}(S(\xi)) \quad, \forall \xi \in \mathbb{T}^{m}
$$

This implies that both

$$
\begin{aligned}
\partial_{t} S & =\operatorname{Jgrad}_{v} H(S), \\
\partial_{t} S & =\Omega \cdot \partial_{\xi} S .
\end{aligned}
$$

We may rewrite these two relations in the form

$$
J^{-1} \Omega \cdot \partial_{\xi} S-\operatorname{grad}_{v} H(S)=0,
$$

and the problem of constructing embedded $m$-dimensional tori which are invariant under the flow of the Hamiltonian PDE is the problem of finding solutions $(S(\xi), \Omega)$ of equation (3.3). This is a bifurcation problem with $m$ parameters, which is in general a small divisor problem.

There have been numerous papers on this subject over the last decade, with several different points of view. Without presenting an exhaustive survey at this point, it is nonetheless useful to point out the principal contributions to date. S. Kuksin [K87, K00] and E. Wayne [W90] first addressed the problem of invariant tori for PDE using extensions of the classical approach of KAM theory, namely the construction of a convergent sequence of canonical transformations. This point of view was pursued in subsequent work, including for example the papers of S. Kuksin and J. Pöschel [KP96] and J. Pöschel [P96]. An alternate and somewhat more direct technique was developed by W. Craig and E. Wayne in [CW93, CW94], which relies on different methods for analysing the linearized operators arising in the problem. This approach was adopted and extended by J. Bourgain in a series of papers [B95, B96, B98]. An overview of these results is given in [B00] and in [C00]. In the 
present article, we describe an approach to the problem of resonant tori based on the latter method. Resonant tori for completely integrable systems arise in parameter families, as can be seen in the unperturbed harmonic oscillator problem. Under perturbation, a family of $m$-dimensional resonant tori will be expected to break up, with only a few $m$-dimensional invariant tori surviving the perturbation. These survivors can be characterized by a variational problem, through which furthermore one can give an estimate on their number. This is the theme of the section below.

\section{(a) the variational problem}

On a formal level, one can restate the equations (3.3) as the Euler - Lagrange equations for a variational problem for mappings of a torus into phase space. Consider the space of such mappings;

$$
S \in X:=\left\{S(\xi): \mathbb{T}^{m} \mapsto \mathcal{H}\right\} .
$$

At this level of discussion we will not specify a particular topology for $X$, realizing however that this is an important consideration in such analysis. Define the action functionals to be

$$
I_{j}(S)=\frac{1}{2} \int_{\mathbb{T}^{m}}\left\langle S, J^{-1} \partial_{\xi_{j}} S\right\rangle_{\mathcal{H}} d \xi, \quad j=1, \ldots m,
$$

whose variation satisfies

$$
\delta_{S} I_{j}=J^{-1} \partial_{\xi_{j}} S .
$$

Consider the average Hamiltonian to be

$$
\bar{H}(S)=\int_{\mathbb{T}^{m}} H(S(\xi)) d \xi,
$$

where evidently

$$
\delta_{S} \bar{H}=\operatorname{grad}_{v} H(S) .
$$

Consider the subvariety of the space $X$ of torus mappings defined by fixing the values of the action integrals;

$$
M_{a}=\left\{S \in X: I_{1}(S)=a_{1}, \ldots I_{m}(S)=a_{m}\right\} \subseteq X .
$$

The equation (3.3) can be viewed as the Lagrange multiplier rule for critical points of $\bar{H}(S)$ restricted to the variety $M_{a}$; suffiently smooth critical points correspond to solutions of equation (3.3) with Lagrange multipliers the components of the frequency vector $\Omega$;

$$
\delta_{S} \bar{H}-\Omega \cdot \delta_{S} I=0 .
$$

It is important to note that both the action functionals $I(S)$ and the average Hamiltonian $\bar{H}(S)$ are invariant under the group action of a torus $\mathbb{T}^{m}$, namely under the transformations

$$
\tau_{\alpha}: S(\xi) \mapsto S(\xi+\alpha), \quad \alpha \in \mathbb{T}^{m},
$$

both $I\left(\tau_{\alpha} S\right)=I(S)$ and $\bar{H}\left(\tau_{\alpha} S\right)=\bar{H}(S)$. Therefore the constraint variety $M_{a}$ is invariant under the action of $\tau_{\alpha}$, and subsequently each critical point is in fact 
a member of a critical orbit by this $m$-torus action. Such considerations will have consequences in counting the number of geometrically distinct solutions of the equations $(3.3)$.

Two questions come to mind at this point.

1. Do critical points exist on $M_{a}$ ? In fact the operators $L(\Omega) S=\Omega \cdot \partial_{\xi} S$ are degenerate on the space of mappings $X$, and their analysis depends in a sensitive way on the diophantine properties of the frequency vector $\Omega$.

2. How to understand the multiplicity of solutions? Each critical point is in fact a member of a critical orbit, but different members of this orbit are geometrically indistinguishable. However, the space $M_{a} / \mathbb{T}^{m}$ possesses nontrivial topology, which implies certain lower bounds on the number of critical orbits of $\tau_{\alpha}$ invariant functionals on $M_{a}$. This is despite the fact that the torus action is not in general a free action, and therefore the set $M_{a} / \mathbb{T}^{m}$ is not in general a smooth variety.

Answers that can be obtained in some cases are that: (1) One uses the Nash - Moser method to overcome the inherent small divisor problem that is present. Our own approach has been to use a method developed in [CW93] which employs Fröhlich - Spencer resolvant estimates for the linearized equations from (3.3). (2) there are well developed methods for counting critical points and critical orbits of functionals on infinite dimensional spaces, which go under the name of Morse Bott theory. In cases of degeneracies, in which the group action is not necessarily a free action, this theory employs the analog of a classical construction of Borel. It is slightly unusual to require the details of this construction in the case of an $m$-dimensional torus action, such as we are encountering, so for the most part the particular details have had to be worked out independently.

\section{(b) the linearized problem}

To start with, the equations of evolution (1.1) linearized around the equilibrium solution $v=(q, p)=0$ are given in terms of the quadratic part of the Hamiltonian

$$
\begin{aligned}
H^{(2)}(v) & =\frac{1}{2} \sum_{k \in \Gamma^{\prime}} \omega(k)\left(\frac{1}{\omega(k)} p(k)^{2}+\omega(k) q(k)^{2}\right) \\
& =\sum_{k \in \Gamma^{\prime}} \omega(k) I(k)
\end{aligned}
$$

where we have represented $v$ in terms of its eigenfunction expansion, as in (2.6). The linearized equations (1.1) around the trivial solution $v=0$ are

$$
\partial_{t}\left(\begin{array}{c}
q(k) \\
p(k)
\end{array}\right)=\left(\begin{array}{cc}
0 & 1 \\
-1 & 0
\end{array}\right)\left(\begin{array}{c}
\omega^{2}(k) q(k) \\
p(k)
\end{array}\right), \quad k \in \Gamma^{\prime} .
$$

Representing mappings $S: \mathbb{T}^{m} \mapsto \mathcal{H}$ of a torus into our phase space,

$$
\begin{aligned}
S & =S(x, \xi)=\sum_{k} S_{k}(\xi) \psi_{k}(x) \\
& =\sum_{j, k} s(j, k) \psi_{k}(x) e^{i j \cdot \xi}, \quad j \in \mathbb{Z}^{m},
\end{aligned}
$$


the equations (3.3) linearized about the zero mapping $S=0$ is written as

$$
\left(\delta_{S}^{2} \bar{H}^{(2)}(0)-\Omega \cdot \delta_{S}^{2} I(0)\right)=\sum_{j, k}\left(\begin{array}{cc}
\omega(k) & i \Omega \cdot j \\
-i \Omega \cdot j & \omega(k)
\end{array}\right)\left(\begin{array}{l}
s_{1}(j, k) \\
s_{2}(j, k)
\end{array}\right) \psi_{k}(x) e^{i j \cdot \xi} .
$$

This represents a decomposition into $2 \times 2$ block diagonal problems, whose eigenvalues are

$$
\mu(j, k)=\omega(k) \pm \Omega \cdot j, \quad j \in \mathbb{Z}^{m}, k \in \Gamma^{\prime}
$$

Chose the bifurcation point to be a frequency vector $\Omega^{0}=\left(\Omega_{1}^{0}, \ldots \Omega_{m}^{0}\right) \in \mathbb{R}^{m}$ which is the solution of $m$-many elementary resonance relations

$$
\omega\left(k_{\ell}\right)-\Omega^{0} \cdot j_{\ell}=0, \quad \ell=1, \ldots m .
$$

This represents a choice of null eigenspace $X_{1} \subseteq X$ within the space of mappings, spanned by the set $\left\{\psi_{k}(x) e^{i j \cdot \xi}: \omega(k) \pm \Omega^{0} \cdot k=0\right\}$. The set of indices satisfying the resonance relations

$$
\omega(k)-\Omega^{0} \cdot j=0
$$

includes at least the set $\left\{\left(j_{\ell}, k_{\ell}\right): \ell=1 \ldots m\right\}$ given as solutions of the elementary resonance relations $(3.16)$, but it is possible that there are many more.

Proposition 3.1. The null space $X_{1}$ is even dimensional, with dimension $2 M \geq$ $2 \mathrm{~m}$. It is possible infinite dimensional, but in any case it is a symplectic subspace of $X$.

The nonresonant case, which is the analog of a simple bifurcation of invariant tori, is when $\operatorname{dim}\left(X_{1}\right)=2 m$. Otherwise $\operatorname{dim}\left(X_{1}\right)=2 M>2 m$, and we say that the case is resonant. The other eigenvalues (3.15) are

$$
\mu(j, k)=\omega(k) \pm \Omega^{0} \cdot j \neq 0,
$$

which typically forms a dense set in $\mathbb{R}$. Accumulating at $\mu=0$, these are the small divisors.

\section{(c) Lyapunov - Schmidt decomposition}

The space $X$ of torus mappings $S: \mathbb{T}^{m} \mapsto \mathcal{H}$ can be decomposed into the null space of the linearized operator (3.14) and its orthogonal complement; $X=X_{1} \oplus X_{2}$. Denote the associated projections by

$$
Q X=X_{1}, \quad P X=(I-Q) X=X_{2} .
$$

We consider the resulting decomposition of mappings;

$$
S=Q S+P S=S_{1}+S_{2} .
$$

The LHS of the nonlinear equation (3.3) is a transformation from the space of mappings $X$ to a range space $Y$, which can also be decomposed into the range $Y_{2}$ of the linearized operator (3.14) and its co-range $Y_{1}$. By abuse of notation we will also denote orthogonal projections onto $Y_{1}$ and $Y_{2}$ respectively, by $Q$ and $P$. The equation to solve (3.3) are equivalent to

$$
\begin{aligned}
& Q\left(J^{-1} \Omega \cdot \partial_{\xi} S-\operatorname{grad}_{v} H(S)\right)=0, \\
& P\left(J^{-1} \Omega \cdot \partial_{\xi} S-\operatorname{grad}_{v} H(S)\right)=0 .
\end{aligned}
$$




\section{(d) critical point theory}

In case $M<+\infty$ the $Q$-equation is finite dimensional, it is in any case called the bifurcation equation. The hard work in our problem at hand is in fact to solve the $P$-equation, for $S_{2}=S_{2}\left(S_{1}, \Omega\right)$ as a function of the parameters $S_{1}$ and $\Omega$. If this is achieved, the variational problem (3.10) is replaced by a reduced variational problem, which is the analog of the one proposed by A. Weinstein [W76] and J. Moser [M79] for periodic orbits of Hamiltonian systems in the resonant case of the Lyapunov center theorem. Define

$$
\begin{aligned}
I_{j}^{1}\left(S_{1}\right) & =I_{j}\left(S_{1}+S_{2}\right) \\
\bar{H}^{1}\left(S_{1}\right) & =\bar{H}\left(S_{1}+S_{2}\right) \\
M_{a}^{1} & =\left\{S_{1} \in X_{1}: I_{j}^{1}\left(S_{1}\right)=a_{j}, j=1 \ldots m\right\}
\end{aligned}
$$

Critical points of $\bar{H}^{1}$ on the subvariety $M_{a}^{1} \subseteq X_{1}$ satisfy

$$
\delta_{S_{1}} \bar{H}^{1}-\Omega \cdot \delta_{S_{1}} I^{1}=0
$$

and these correspond to solutions of the $Q$-equation (3.17). This fact focuses our attention on the reduced constraint variety $M_{a}^{1}$ itself. The solution of the $P$-equation is equivariant with respect to the group action $\tau_{\alpha}$, and it follows that the reduced functionals $I_{j}^{1}\left(S_{1}\right)$ and $\bar{H}^{1}\left(S_{1}\right)$ are invariant with respect to the $\mathbb{T}^{m}$ action $\tau_{\alpha}$. By its topology alone we can deduce a lower bound for the number of distinct critical $\mathbb{T}^{m}$ orbits of $\bar{H}^{1}\left(S_{1}\right)$ on $M_{a}^{1}$. This answer, which is still partially conjectural, is that in fact that, given the $m$-dimensional action parameter $a$, there exist integers $p_{1}, \ldots p_{m}$ such that $M_{a}^{1}$ is a product of $m$-many odd dimensional spheres,

$$
M_{a}^{1} \simeq \otimes_{j=1}^{m} S^{2 p_{j}-1} .
$$

The dimensions $p_{j}=p_{j}(a)$ satisfy $\sum_{j} p_{j}=m$. They are not constant, but change as a crosses certain singular homogeneous varieties. This process of transfer of dimension from some spheres to other ones is an example of symplectic surgery. For each choice of the sequence $\left(p_{j}(a)\right)_{j=1}^{m}$, the $\mathbb{T}^{m}$ equivariant Morse-Bott inequalities give a lower bound on the number of critical points of sufficiently nondegenerate $\mathbb{T}^{m}$-invariant functionals on $M_{a}^{1}$. A simple answer which is uniform under all choices of $\left(p_{j}\right)_{j=1}^{m}$ is the following.

Theorem 3.2. The number of distinct critical $\mathbb{T}^{m}$ orbits of $\bar{H}^{1}$ on $M_{a}^{1}$ is bounded below:

$$
\#\left\{\text { critical orbits of } \bar{H}^{1}\right\} \geq(M-m+1) .
$$

This lower bound corresponds to the simplest estimate given by equivariant cohomology on $M_{a}^{1} / \mathbb{T}^{m}$. In fact the estimate holds independently of the orbittransversal nondegeneracy condition usually encountered in Morse-Bott theory, using multiplicative rather than additive techniques.

It is useful to check this inequality in the endpoint cases. Set $m=1$ for periodic orbits, and the number of resonant frequencies $M>1$ arbitrary. Then $M_{a}^{1} \simeq S^{2 M-1}$ and $M_{a}^{1} / \mathbb{T}^{1} \simeq \mathbb{C} P(M-1)$, a (possibly weighted) complex projective space. The Morse inequalilies in this case imply that the number of distinct critical orbits of 
$\mathbb{T}^{1}$-invariant functions on $M_{a}^{1}$ is at least $M$. This corresponds to the case of the Weinstein - Moser theorem on the resonant Lyapunov center theorem.

Now set $m=M$, the case of quasiperiodic motion on a nonresonant $m$-torus. In this case,

$$
M_{a}^{1} \simeq \otimes_{j=1}^{m} S^{1}
$$

which is a product of circles. Under the group action by $\mathbb{T}^{m}$, we have $M_{a}^{1} / \mathbb{T}^{m} \simeq *$, a point, corresponding to having one critical $m$-torus. This is a classical KAM torus. In recent work on doubly periodic surface water wave patterns which are resonant, the case $m=2, M \geq 2$ occurs, and the estimate on the number of geometrically distinct doubly periodic solutions is that $\#\{$ critical orbits $\} \geq(M-1)$.

\section{Estimates of the linear problem}

The critical issue is to solve the $P$-equation (3.18) for the component of an $m$-torus embedding $S_{2}=S_{2}\left(S_{1}, \Omega\right)$. This is typically a small divisor problem, which we address with a Nash - Moser implicit function theorem. Central to the workings of the method is an analysis of the linearized equation. The problem (3.18) is for an $m$-torus mapping, projected by $P$. With $S_{1}=0$ and then differentiating with respect to $S_{2} \in P \mathcal{H}$ at the point $S_{2}=0$, the linearization of (3.18) is

$$
P\left(\delta_{S_{2}}^{2} \bar{H}(0)-\Omega \cdot \delta_{S_{2}}^{2}(0)\right) P V=G,
$$

where $P V=V$ and $P G=G$. In coordinates given by the eigenfunction expansion, equation (4.1) is expressed as

$$
\left(\begin{array}{cc}
\omega(k) & i \Omega \cdot j \\
-i \Omega \cdot j & \omega(k)
\end{array}\right)\left(\begin{array}{l}
v_{1}(j, k) \\
v_{2}(j, k)
\end{array}\right)=\left(\begin{array}{l}
g_{1} \\
g_{2}
\end{array}\right), \quad j \in \mathbb{Z}^{m}, k \in \Gamma^{\prime} .
$$

It is seen that the spectrum of the operator on $P \mathcal{H}$ is the set $\{\mu(j, k)=\omega(k)-\Omega \cdot j \neq$ $\left.0:(j, k) \in \mathbb{Z}^{m} \oplus \Gamma^{\prime}\right\}$. Typically this is a dense set of $\mathbb{R}$, the eigenvalues accumulating at zero constituting the small denominators of the problem. The Newton iteration scheme that is used in the Nash - Moser technique requires inversion of the linearized operator about an approximate torus embedding $S^{0}=S_{1}+S_{2}^{0}$, more than just the linearization about zero. That is, for $V=P V \in X_{2}$ we are drawn to the analysis of the operator

$$
\begin{aligned}
& P\left(\delta_{S_{2}}^{2} \bar{H}\left(S_{1}+S_{2}^{0}\right)-\Omega \cdot \delta_{S_{2}}^{2}\left(S_{1}+S_{2}^{0}\right)\right) V \\
& =P\left(\operatorname{diag}_{2 \times 2}\left(\begin{array}{cc}
\omega(k) & i \Omega \cdot j \\
-i \Omega \cdot j & \omega(k)
\end{array}\right)+W\left((j, k),\left(j^{\prime}, k^{\prime}\right)\right)\right) V=G .
\end{aligned}
$$

The additional off-diagonal terms $W$ come from the linearization of nonlinear terms of the equation (3.3). They represent a perturbation of a $2 \times 2$-block diagonal operator with dense spectrum. Estimates of this linearized operator are obtained by an adaptation of the method of J. Fröhlich and T. Spencer [FS83] using resolvant estimates and block decompositions of the lattice of indices of eigenmodes $\{y:=$ $\left.(j, k) \in \mathbb{Z}^{m} \oplus \Gamma^{\prime}\right\}$. We work with a scale of Hilbert spaces of sequences on this lattice, which I will not specify precisely in this note. However we will denote the appropriate operator norm for this scale of spaces by $|\cdot|_{o p}$. A more detailed account 
of the analytic procedure appears in [CW93, C00] and [B95, B96, B98]. One first separates the lattice sites into the singular and the regular regions.

Definition 4.1. A lattice site $y=(j, k) \in \mathbb{Z}^{m} \oplus \Gamma^{\prime}$ is $d_{0}$-singular for the frequency $\Omega$ when

$$
|\omega(k) \pm \Omega \cdot j|<d_{0} .
$$

Otherwise, the lattice site $y$ is regular.

Let $B$ be a subset of the lattice $\mathbb{Z}^{m} \oplus \Gamma^{\prime}$, and let the orthogonal projection within $\mathcal{H}$ onto the subspace spanned by sequences $(s(y): y \in B)$ be denoted by $P_{B}$. The restriction of an operator $H$ to the subset $P_{B} \mathcal{H}$ is denoted by $H_{B}=P_{B} H P_{B}$.

Proposition 4.1. For a set $A \subseteq \mathbb{Z}^{m} \oplus \Gamma^{\prime}$ consisting of regular lattice sites, and for $|W|_{o p}<d_{0} / 2$, then the linearized operator (4.3) is invertible, and satisfies the estimate

$$
\left|P\left(\delta_{S_{2}}^{2} \bar{H}\left(S_{1}+S_{2}^{0}\right)-\Omega \cdot \delta_{S_{2}}^{2}\left(S_{1}+S_{2}^{0}\right)\right)_{A}^{-1}\right|_{O p} \leq \frac{4}{d_{0}} .
$$

Following [FS83], resolvant estimates are used in order to add the singular sites of the lattice $\mathbb{Z}^{m} \oplus \Gamma^{\prime}$ in the construction of the inverse operator $P\left(\delta_{S_{2}}^{2} \bar{H}\left(S_{1}+S_{2}^{0}\right)-\right.$ $\left.\Omega \cdot \delta_{S_{2}}^{2}\left(S_{1}+S_{2}^{0}\right)\right)^{-1}$. These will be quantified in the iteration scheme, over enlarging subsets of the lattice $\mathbb{Z}^{m} \oplus \Gamma^{\prime}$ which grow to exhaust the lattice. Indeed, take regions $B_{n}=\left\{y=(j, k) \in \mathbb{Z}^{m} \oplus \Gamma^{\prime}:|y| \leq R_{n}:=L_{0} 2^{n}\right\}$. Our ability to carry through the resolvant estimates depend upon two properties of the frequencies $\Omega \in \mathbb{R}^{m}$ and the linearized operator

$$
P\left(\delta_{S_{2}}^{2} \bar{H}\left(S_{1}+S_{2}^{0}\right)-\Omega \cdot \delta_{S_{2}}^{2}\left(S_{1}+S_{2}^{0}\right)\right):=D(\Omega)+W .
$$

property 1. nonresonance. Suppose that $y=(j, k)$ and $y^{\prime}=\left(j^{\prime}, k^{\prime}\right)$ in $\mathbb{Z}^{m} \oplus \Gamma^{\prime}$ are two singular sites within the region $B_{n+1} \backslash B_{n}$. Then both

$$
\begin{aligned}
& d_{n}<|\omega(k)-\Omega \cdot j|<d_{0} \\
& d_{n}<\left|\omega\left(k^{\prime}\right)-\Omega \cdot j^{\prime}\right|<d_{0} .
\end{aligned}
$$

The lower bound is adapted in the iteration scheme, $d_{n}=d_{0} \exp (-\beta n)$, and the frequency parameters $\Omega$ for which (4.7) is violated are excised. This is at the origin of the Cantor set over which the resulting existence theorem will hold.

property 2. separation. Suppose that $y, y^{\prime}$ are two singular sites within $B_{n+1} \backslash B_{n}$. We ask that either

(i). $\operatorname{dist}\left(y, y^{\prime}\right)<R_{n}^{\alpha}$, or else

(ii). $\operatorname{dist}\left(y, y^{\prime}\right) \gg R_{n}^{\gamma}$.

Typically the choice must be that $0<\alpha \ll \gamma<1$. In words, the requirement is that within the $n^{t h}$ annulus $B_{n+1} \backslash B_{n}$, the singular sites can be divided into clusters, with slow growth of the cardinality of each cluster, namely $R_{n}^{\alpha m}$, and with large distances $R_{n}^{\gamma}$ between clusters.

It is principally in this second property that the specific details of the particular partial differential equation and its nonlinearity and dispersion relation come into 
the analysis in an important way. In particular, the Schrödinger equation in one space dimension has singular sites which are automatically well separated, due to the nondegeneracy of the dispersion relation. The nonlinear wave equation in one dimension achieves the required degree of separation throug a diophantine condition on the frequency $\Omega^{0}$. Problems in higher space dimensions address the two conditions with increasingly sophisticated techniques, which can be seen in more detail in [B95, B96]. It is however beyond the scope of the present article to pursue this in any further detail.

Acknowledgements: This research has been supported in part by the Canada Research Chairs Program, the NSERC under operating grant \#238452, and the NSF under grant \#DMS-0070218.

\section{References}

[BB97] T.B. Benjamin and T.J. Bridges. Reappraisal of the Kelvin-Helmholtz problem. I. Hamiltonian structure. J. Fluid Mech. 333 pp. 301-325, (1997).

[B95] J. Bourgain. Construction of periodic solutions of nonlinear wave equations in higher dimension. Geom. Funct. Anal. 5 (1995), no. 4, pp. 629-639.

[B96] J. Bourgain. Construction of approximative and almost periodic solutions of perturbed linear Schrödinger and wave equations. Geom. Funct. Anal. 6 (1996), no. 2, pp. 201-230.

[B98] J. Bourgain. Quasi-periodic solutions of Hamiltonian perturbations of 2D linear Schrödinger equations. Ann. of Math. (2) 148 (1998), no. 2, pp. 363-439.

[B00] J. Bourgain. Problems in Hamiltonian PDE's. GAFA 2000 (Tel Aviv, 1999). Geom. Funct. Anal. (2000), Special Volume, Part I, pp. 32-56.

[C00] W. Craig. Problèmes de petits diviseurs dans les équations aux dérivées partielles. (French) [Small divisor problems in partial differential equations] Panoramas et Synthèses 9. Société Mathématique de France, Paris, 2000. viii+120 pp. ISBN: 285629-095-7

[CGK03] W. Craig, P. Guyenne \& H. Kalisch. A new model for large amplitude long internal waves. C. R. Acad. Sci. Paris 332 (2004), pp. 525 - 530.

[CGK04] W. Craig, P. Guyenne \& H. Kalisch. Hamiltonian long wave expansions for free surfaces and interfaces. submitted to Commun. Pure Applied Math. (2004).

[CS93] W. Craig and C. Sulem. Numerical simulation of gravity waves. J. Comp. Phys.108, pp. 73-83, (1993).

[CW93] W. Craig and C.E. Wayne. Newton's method and periodic solutions of nonlinear wave equations. Commun. Pure Applied Math. XLVI pp. 1409-1501, (1993).

[CW94] W. Craig and C.E. Wayne. Periodic solutions of nonlinear Schrdinger equations and the Nash-Moser method. Hamiltonian mechanics (Toruń, 1993), pp. 103-122, NATO Adv. Sci. Inst. Ser. B Phys., 331, Plenum, New York, (1994).

[FS83] J. Fröhlich and T. Spencer. Absence of diffusion in the Anderson tight binding model for large disorder or low energy. Comm. Math. Phys. 88 (1983), no. 2, pp. $151-184$.

[H10] J. Hadamard. Leçons sur le calcul des variations. Hermann, Paris, 1910; paragraph $\S 249$.

[H16] J. Hadamard. Sur les ondes liquides. Rend. Acad. Lincei 5, (1916) no. 25, pp. 716-719.

[K87] S. B. Kuksin. Hamiltonian perturbations of infinite-dimensional linear systems with imaginary spectrum. (Russian) Funktsional. Anal. i Prilozhen. 21 (1987), no. 3, pp. 22-37, 95. 
[K00] S. B. Kuksin. Analysis of Hamiltonian PDEs. Oxford Lecture Series in Mathematics and its Applications, 19. Oxford University Press, Oxford, 2000. xii+212 pp. ISBN: 0-19-850395-4

[KP96] S. Kuksin and J. Pöschel. Invariant Cantor manifolds of quasi-periodic oscillations for a nonlinear Schrödinger equation. Ann. of Math. (2) 143 (1996), no. 1, pp. 149-179.

[M79] J. Moser. Periodic orbits near an equilibrium and a theorem by Alan Weinstein. Comm. Pure Appl. Math. 29 (1976), no. 6, pp. 724-747.

[P96] J. Pöschel. A KAM-theorem for some nonlinear partial differential equations. Ann. Scuola Norm. Sup. Pisa Cl. Sci. (4) 23 (1996), no. 1, pp. 119-148.

[W90] E. Wayne. Periodic and quasi-periodic solutions of nonlinear wave equations via KAM theory. Comm. Math. Phys. 127 (1990), no. 3, pp. 479-528.

[W76] A. Weinstein. Normal modes for nonlinear Hamiltonian systems. Invent. Math. 20 (1973), pp. 47-57.

[Z68] V.E. Zakharov. Stability of periodic waves of finite amplitude on the surface of a deep fluid. J. Appl. Mech. Tech. Phys. 9:190-194, 1968. 